\title{
Studying growth kinetics of microbial populations using information technology. Solving the Cauchy problem
}

\author{
Marina A. Nikitina ${ }^{1, *}$, and Irina M. Chernukha ${ }^{2}$ \\ ${ }^{1}$ V.M. Gorbatov Federal Research Center for Food Systems of RAS, Center of Economic and \\ Analytical Research and Information Technologies, 109316 Moscow, Russia \\ ${ }^{2}$ V.M. Gorbatov Federal Research Center for Food Systems of RAS, Experimental clinic-laboratory \\ "Biologically active substances of an animal origin", 109316 Moscow, Russia
}

\begin{abstract}
The possibilities of information technologies in the study of growth dynamics and development of microbial populations have been shown. In the $\mathrm{R}$ programming language in the Jupyter Notebooks environment, a direct kinetic problem has been solved. Kinetic regularities of growth of microbial populations under periodic cultivation have been considered within the framework of an approximation based on numerical integration of velocity equations. The one-step Runge-Kutta method of the fourth order of accuracy has been used as a method for solving a differential equation with initial conditions (Cauchy problem). Initial conditions of the problem were: the number of time steps $n=10,000$; initial substrate concentration $\mathrm{S}_{0}=1$; the initial concentration of microorganisms has been considered in four variants: $\mathrm{M}_{0}=0.01, \mathrm{M}_{0}=0.05, \mathrm{M}_{0}=0.1, \mathrm{M}_{0}=0.2$, which correspond to $1 \%, 5 \%, 10 \%, 20 \%$ of the inoculum density accordingly; affinity ration of the substrate to microorganisms $\mathrm{Ks}=0.5$. The use of modern information technologies in the analysis of microbial growth patterns is mainly determined by the capabilities of personal computers, software environments and shells. The potential of modern software in the implementation of applied engineering and research problems in solving ordinary differential equations describing the development and course of the microbial process over time has been presented.
\end{abstract}

\section{Introduction}

The basic part of microbiology is related to microbial growth kinetics, i.e. determining the ratio between the growth rate of a specific microbial population and the substrate concentration.

To verify any theory, a sufficient amount of good-quality experimental data is needed. Conducting a large number of experiments is not always possible. In this case, methods of mathematical and simulation (computer) modeling are applicable. Metadata of articles

\footnotetext{
* Corresponding author: nikitinama@yandex.ru
} 
published over the past decades show that simulation is increasingly used in the study and perception of microbial cell growth mechanisms.

Using simulation models that represent the virtual flow of a process, it is possible to solve a number of problems from studying the behavior of a complex system with a given set of characteristics when changing internal and/or external parameters to solving optimization problems.

This approach 1) allows conducting a fundamental study of complex processes, taking into account the diversity of factors, assessing the growth kinetics of not only one, but also mixed populations; 2) justifying technological modes for controlling the expiration of the microbiological process.

There are currently quite many publications on mathematical modeling of microbial processes: models of biomass accumulation; models of accumulation of other products of microbial activity; models that take into account the age structure of the population; models of autoselection; models of adaptation of microbial communities [1-14]. The first works on optimization and modeling of microorganisms in the USSR are associated with the names of such scientists as V. Biryukov, M. Kantere, Yu. Malashenko, G. Yablonsky, and others [1517]. It is worth noting that G. Yablonsky was solving the inverse kinetic problem in his works.

Among the approaches to the study of the growth kinetics of microbial populations, the most widely used approach is based on numerical integration. First, integration can be performed with any accuracy that significantly exceeds the accuracy of the experimental study. Second, numerical explicit functions that link experimentally determined quantities with time can be obtained.

The application of modern high-performance computers and information technologies, including in solving the Cauchy problem, is particularly indisputable when studying the dynamics of complex microbial processes (processes in mixed and symbiotrophic cultures). Analytical integration of velocity equations for systems of this type becomes impossible.

The purpose of this paper is to show the solution of a direct kinetic problem (for example, the solution of the Cauchy problem) using simulation and information technologies.

\section{Methods}

Numerical integration of differential equations allows solving most applied problems, including equations of mathematical physics, describing the dynamics of growth and development of microbial populations, etc., which change over time and/or space. One of the solutions for the given initial conditions is related to the Cauchy problem.

One-step numerical methods are used to solve the Cauchy problem. The algorithm for finding the $i+1$-th (next) point on the $y=f(t)$ curve is related only to the information about the previous step. These methods include the Euler method and its modifications, as well as improved Runge-Kutta method. An important advantage of the 4th-order Runge-Kutta method is the ability to change the integration step at any stage of the calculation, subject to compliance with the specified accuracy. These methods are based on the decomposition of $y$ into the Taylor series in the vicinity of $t_{0}$ with the retention of a finite number of terms of series [18].

The software implementation was performed in the Jupyter Notebooks environment in the $\mathrm{R}$ programming language [19-20]. 


\section{Results}

Differential equations of the growth rate (1) and substrate flow rate (2) in dimensionless variables have the following form:

$$
\begin{gathered}
\frac{d m}{d r}=\frac{m s}{K_{S} / s_{0}+S} \\
\frac{d s}{d r}=-\frac{m s}{K_{s} / S_{0}+S} \\
s=\frac{S}{S_{0}} ; m=\frac{M}{M_{\infty}-N_{\infty}} ; p=\frac{P}{P_{\infty}} ; \tau=\mu_{m} t
\end{gathered}
$$

where $\mathrm{s}$ is the substrate concentration in dimensionless quantities; $\mathrm{m}$ is the concentration of biomass (microorganisms) in dimensionless quantities; $\mathrm{S}$ is the concentration of substrate in physical (dimensional) quantities; $\mathrm{M}$ is the concentration of biomass (microorganisms) in physical (dimensional) quantities; $S_{0}$ is the initial substrate concentration; $M_{0}$ is the initial concentration of biomass (microorganisms); $\mathrm{K}_{\mathrm{s}}$ is the constant of affinity of substrate to biomass (to microorganism); $\mathrm{M}_{\infty}$ is the limiting quantity of biomass (microorganisms) and product formed at infinite great time of the process. $\mathrm{M}_{\infty}$ is related to initial conditions: substrate and biomass (microorganisms) concentrations.

The following data were taken as initial data of the problem: periodic cultivation; number of time steps $n=10000$; initial substrate concentration $S_{0}=1$; the initial concentration of microorganisms has been considered in four variants: $\mathrm{M}_{0}=0.01, \mathrm{M}_{0}=0.05, \mathrm{M}_{0}=0.1, \mathrm{M}_{0}=0.2$, which correspond to $1 \%, 5 \%, 10 \%, 20 \%$ of the inoculum density accordingly; affinity ration of the substrate to microorganisms $\mathrm{K}_{\mathrm{s}}=0.5$. Other factors were not taken into account in the calculation. The Cauchy problem was solved using the Runge-Kutta method of the fourth order of accuracy in the R programming language in the Jupyter Notebooks environment. The listing fragment is shown in Fig. 1.

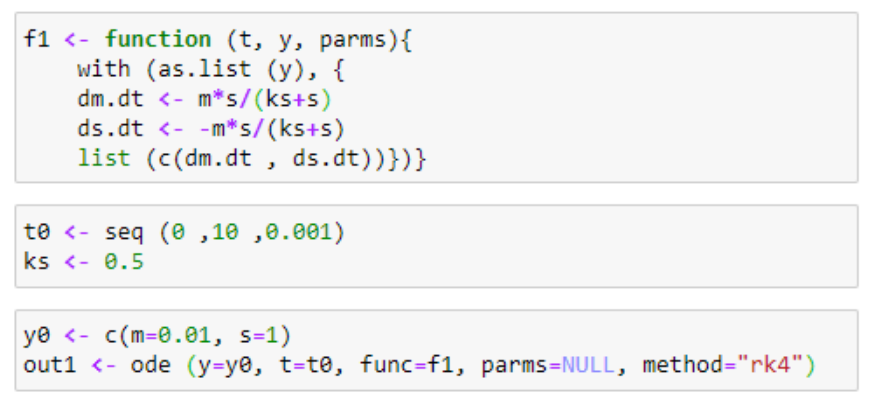

Fig. 1. Software code listing

Integrated equations (1) and (2) with initial conditions gave the following results (table 1).

Table 1. Fragment of the solution (calculation) result.

$\begin{array}{ccc}\text { time } & \mathbf{m} & \mathbf{s} \\ 0.000 & 0.01000000 & 1.0000000 \\ 0.001 & 0.01000667 & 0.9999933 \\ 0.002 & 0.01001334 & 0.9999867\end{array}$




\begin{tabular}{rrr} 
time & \multicolumn{1}{c}{$\mathbf{m}$} & \multicolumn{1}{c}{$\mathbf{s}$} \\
0.003 & 0.01002002 & 0.9999800 \\
0.004 & 0.01002670 & 0.9999733 \\
$\ldots$ & $\ldots$ & $\ldots$ \\
\hline 9.971 & 1.007990 & 0.002009697 \\
\hline 9.972 & 1.007994 & 0.002005666 \\
\hline 9.973 & 1.007998 & 0.002001643 \\
\hline$\ldots$ & $\ldots$ & \\
\hline 9.999 & 1.008100 & 0.001899809 \\
\hline 10.000 & 1.008104 & 0.001895997 \\
\hline
\end{tabular}

Along with the table values, dependences of the biomass concentration and initial substrate on time for systems with different amounts of the introduced substrate have been obtained (Fig. 2-3).

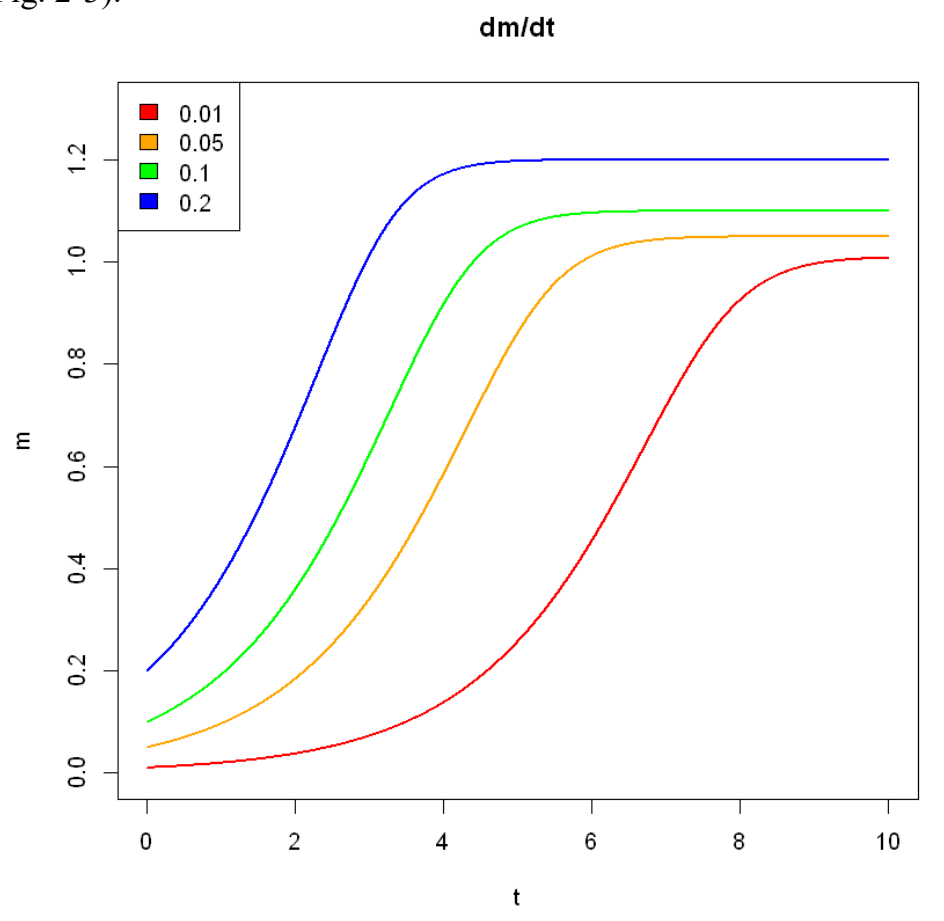

Fig. 2. Diagram of the microorganism concentration dependencies on time in dimensionless units for systems with $1 \%$ (red line), $5 \%$ (yellow line), $10 \%$ (green line), $20 \%$ (blue line) of the introduced substrate 


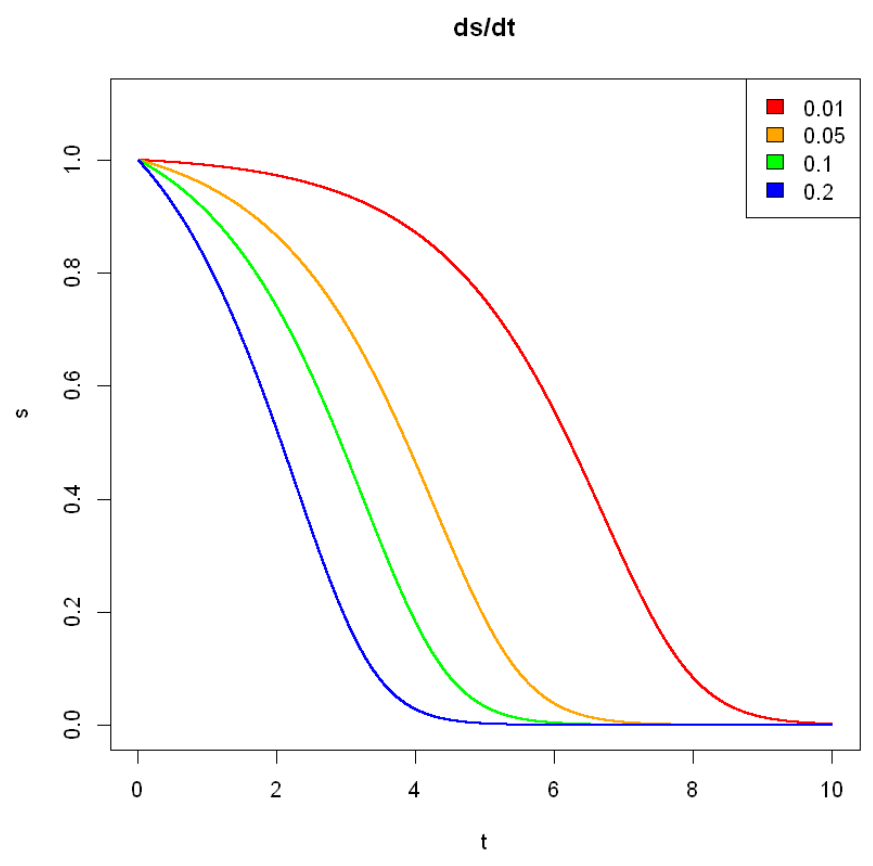

Fig. 3. Diagram of the source substrate dependencies on time in dimensionless units for systems with $1 \%$ (red line), $5 \%$ (yellow line), $10 \%$ (green line), $20 \%$ (blue line) of the introduced substrate

Comparison and analysis of graphical dependencies obtained as a result of the calculation (Fig. 2-3) show that the speed of the process depends solely on the amount of substrate introduced. As can be seen from figures, when sowing with 0.01 ( $1 \%$, corresponds to the red line) of the culture, the substrate consumption during the process corresponds to 6.2 units at the $t$ coordinate. When sowing with $0.2(20 \%$, corresponds to the blue line $)-2.1$. units at the t coordinate. The reduction occurs only due to the concentration of the introduced substrate.

\section{Conclusion}

When conducting scientific research with the use of mathematical apparatus it is necessary to use modern information technology. One of its tools is the simulation modeling. The solution of an ordinary differential equation with initial conditions (Cauchy problem) in the Jupyter Notebooks environment in the R programming language is shown on the example of forecasting the dynamics of growth and development of microbial populations. Visual representation in the form of tabular and graphical data will help the researcher not only in studying the process, establishing patterns and predicting results, but also in making informed decisions.

This article is published as part of scientific research theme No. 0585-2019-0008 under the state assignment of the federal state budgetary scientific institution 'V.M. Gorbatov Federal Research Centre for Food Systems' of RAS.

\section{References}

1. K. Olsson-Francis, N. K. Ramkissoon, M. C. Macey, V. K. Pearson, S. P. Schwenzer, D. N. Johnson, J. of microbiological methods 172, (2020) 
2. S. M. Clifford, J. of geophysical research-planets 98(E6), 10973 (1993)

3. H. D. Hu, K. W. Liao, W. M. Xie, J. F. Wang, B. Wu, H. Q. Ren, Water research 174 (2020)

4. Y. Bareha, R. Girault, S. Guezel, J. Chakera, A. Trémierac, Water research 154, 298 (2019)

5. X. Hu, K. Wisniewski, K. Czerwionka, Q. Zhou, L. Xie, J. Makinia, Environmental science and technology 50, 1887 (2016). DOI: 10.1021/acs.est.5b04849

6. K. Liao, H. Hu, S. Ma, H. Ren, Water research 159, 397 (2019). DOI: 10.1016/j.watres.2019.04.049

7. X. Lu, T. D. S. Pereira, H. E. Al-Hazni, J. Majtacz, Q. Zhou, L. Xie, J. Makinia, Environmental science and technology 52, 2800 (2018)

8. H. Simsek, Environmental technology 37, 2879 (2016). DOI: 10.1080/09593330.2016.1167964

9. N. K. Kalita, S. M. Bhasney, A. Kalamdhad, V. Katiyar, Journal of Environmental Management 261 (2020). DOI: 10.1016/j.jenvman.2020.110211

10. V. Janes-Bassett, J. Davies, E. C. Rowe, E. Tipping, Science of The Total Environment 714 (2020). DOI: 10.1016/j.scitotenv.2020.136599

11. G. G. Zhang, J. Huang, M. Q. Jia, F. H. Liu, Y. H. Yang, Z. W. Wang, G. D. Han, Soil Science Society of America J. 83, 1683 (2019)

12. S. S. Hewavitharana, E. Klarer, A. J. Reed, R. Leisso, B. Poirier, L. Honaas, D. R. Rudell, M. Mazzola, Frontiers in Microbiology 10 (2019)

13. J. C. Davis, J. P. Shannon, M. J. Van Grinsven, N. W. Bolton, J. W. Wagenbrenner, R. K. Kolka, T. G. Pypker, Biogeochemistry 145, 275 (2019). DOI: 10.1007/s10533-01900604-2

14. H. Abe, K. Koyama, S. Kawamura, S. Koseki, Food Microbiology 82, 436 (2019)

15. V. V. Biryukov, M. V. Kantere, Optimizaciya periodicheskih processov kul'tivirovaniya mikroorganizmov [Optimization of periodic processes of microbial cultivation] (Nauka, Moscow, 1985)

16. Yu. R. Malashenko, F. V. Muchnik, V. A. Romanovskaya, Yu. S. Sadovnikov, Matematicheskie modeli i EVM v mikrobiologicheskoj praktike [Mathematical models and computers in microbiological practice] (Naukova Dumka, Kiev, 1980)

17. G. S. Yablonskij, S. I. Spivak, Matematicheskie modeli himicheskoj kinetiki [Mathematical models of chemical kinetics] (Znanie, Moscow, 1977)

18. L. I. Turchak, Osnovy chislennyh metodov [Fundamentals of numerical methods] (Nauka, Moscow, 1987)

19. H. Wickham, G. Grolemund, $R$ for Data Science: Import, Tidy, Transform, Visualize, and Model Data (O'Reilly Media, Inc., Sebastopol, 2016)

20. R. I. Kabacoff, $R$ in Action: Data analysis and graphics with $R$ (Manning, Shelter Island, 2011) 